(C) 2013 IEEE. Personal use of this material is permitted. Permission from IEEE must be obtained for all other uses, in any current or future media, including reprinting/republishing this material for advertising or promotional purposes, creating new collective works, for resale or redistribution to servers or lists, or reuse of any copyrighted component of this work in other works. 


\title{
Enhancing the Performance of Electro-Optical Heterodyne Receivers Using Gain Switched Photonic Local Oscillators
}

\author{
Cristina de Dios, A. Rubén Criado, Student Member, IEEE, Estefanía Prior, and Pablo Acedo, Member, IEEE
}

\begin{abstract}
The performance of a Gain Switched (GS) optical source used as a Photonic Local Oscillator (PLO) in an Electro-Optical (EO) heterodyne receiver is experimentally evaluated and compared to the use of a traditional linearly modulated PLO. The EO heterodyne receiver used is based on an Ultra Nonlinear Semiconductor Optical Amplifier (XN-SOA) that provides a compact, cost effective and energy efficient receiver with a remote distribution of both PLO and Intermediate Frequency (IF) signals and a direct 50-ohms input for the Radiofrequency (RF) component. The comparison between both GS and linearly modulated PLO is made in terms of the conversion efficiency (conversion ratio); linearity (1-dB compression point) and noise (integrated RMS timing jitter). The analysis results in a significant improvement of the evaluated figures of merit when using a Gain Switched PLO.
\end{abstract}

Index Terms-Electro-optical receiver, gain switching, heterodyne receiver, photonic local oscillator.

\section{INTRODUCTION}

$\mathbf{P}$ ROCESSING of radiofrequency (RF) signals in the optical domain has become one of the most active research fields [1], [2]. This is mainly due to the advantages that the optical approach offers, such as high bandwidth capabilities, Electromagnetic Interference (EMI) immunity, extremely low transmission losses when using optical fibers and the possibilities of including signal processing features, like True Time Delay (TTD) [3]. Another key advantage is the availability of a wide variety of high performance, compact and low cost photonic devices in the telecom wavelength range, suitable for their use in Microwave Photonics and Radio over Fiber (RoF) systems [1], [2].

Among the variety of new technologies that have been reported [1], [2], the investigation of photonic heterodyne receivers in the $\mathrm{GHz}$ range is an important field of study, for which the development of an optoelectronic mixer is a key element. Several optical mixing schemes have been already introduced based on elements as Mach-Zehnder modulators

This work was supported in part by the Spanish Ministry of Science and Technology through the project TEC2009-14525-C02-02. The work of A R. Criado has been supported by the Spanish Ministry of Science and Technology under the FPI Program, Grant \# BES2010-030290.

The authors are with the Electronics Technology Department, Universidad Carlos III de Madrid, Leganés, Madrid 28911, Spain (e-mail: cdios@ing.uc3m. es).
[4], electro-absorption modulators [5] or dual-mode monolithic laser sources [6]. The photonic heterodyne receivers can be combined with the use of remote photonic distribution of Local Oscillators (LO) and Intermediate Frequencies (IF) architectures that are becoming common place in the last years [7]. Combination of such architectures and a photonic receiver able to perform directly the mixing of the LO (distributed over optical fiber) and the RF with high sensitivity [7] will lead to flexible and scalable solutions for RoF heterodyne receivers.

Recently, Semiconductor Optical Amplifier (SOA) have been also proposed as optoelectronic mixers [8]-[11]. They are versatile, compact and reliable low cost devices. The mixing process using a SOA can be accomplished in all optical [11] or Electro-Optical (EO) configuration. In the EO mixing, only one of the electrical signals, the local oscillator (LO) or the radio-frequency $(\mathrm{RF})$, is modulated on an optical carrier. The other electrical signal modulates the SOA bias voltage [8], [10]. Both downconversion [10] and upconversion [9] have been reported, considering an electrical LO.

The most straightforward Photonic LO (PLO) to be used in such architectures is based on the linear amplitude modulation of the power emitted by a diode laser, either using direct modulation or via an external modulator, being this later approximation polarization dependent and more complex in terms of component count. In addition to these two, if special operating regimes of the diode lasers are also considered, other ways of generating a PLO can be accomplished. The use of the Gain Switching (GS) technique is one of them [12], [13]. It is based on the direct modulation of diode lasers under specific bias current, modulation and frequency conditions to obtain picosecond optical pulses in the temporal domain. In the frequency domain, it shows a comb-like spectrum, different to the one offered by a linear PLO. Using this type of PLO, a good conversion efficiency has been reported in a EO heterodyne configuration [8].

In this work we present a detailed study of a scheme for EO downconversion where the PLO and the retrieved IF are remotely distributed over fiber to a single Commercial Off the Shelf (COTS) Ultra Nonlinear SOA (XN-SOA) that acts as a mixer device [8]. Several figures of merit are evaluated to study the quality of the detection such as the conversion efficiency, the linearity ( $1 \mathrm{~dB}$ compression factor) and the phase noise influence through the root mean square (RMS) integrated jitter. In order to provide a reference for one of the potential applications of this EO receiver, this last figure of merit is evaluated according to the jitter requirements of ITU-T G.709 standard for Optical Transport Networks (OTN) [14], [15]. The dependence 


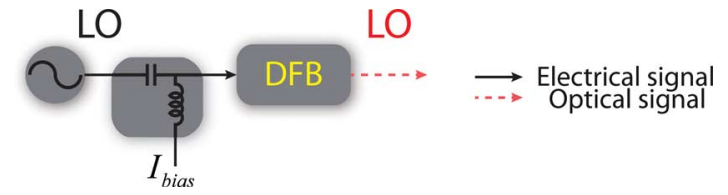

Fig. 1. Optical spectra of the DFB under linear and GS modulation.

of the performance of the $\mathrm{EO}$ heterodyne receiver with the mixer element (XN-SOA) operating conditions (i.e., bias current) and with the RF frequency is also considered.

All these parameters are evaluated considering two PLO scenarios. The first one is a linear directly modulated Distributed Feedback (DFB) laser diode and the second is a GS pulsed optical source, based on the same device, and emitting the same average optical power. The two schemes are polarization independent and are applied to the same EO heterodyne receiver. Results show that under the figures of merit considered, the GS PLO offers better results, with a conversion ratio up to $-15 \mathrm{~dB}$, a linear behavior in the whole analyzed frequency range and an enhanced RMS integrated jitter performance.

\section{Photonic LocAl OSCILlator}

As mentioned in the introduction, the influence of the PLO scheme used in an EO mixing architecture is one of the main objectives of this work. In this sense, we have considered two possibilities: a linear PLO, which is the commonly used LO scheme, and a GS based PLO. In the next paragraphs we discuss the use of the Gain Switching technique in the framework of PLO for EO mixing.

Gain Switching (GS) is a direct and straightforward technique typically used to obtain picosecond optical pulses from semiconductor laser sources. Such gain switched optical pulses inherit many advantages that compact semiconductor devices provide, chiefly the frequency tunability, possibility for high repetition rates and their low cost nature [16]-[18]. Although it can be applied to any diode laser (Commercial Off the Shelf, COTS, devices), it is more advantageous to use it in single mode diode lasers, since they will be less affected by dispersion and nonlinearities when propagating through an optical fiber. Also, studies have shown that they offer better quality pulsed regime [13]. Hence, for our study of a local oscillator based on a GS optical source, we will consider a COTS DFB device emitting at 1550 nm (QPhotonics 1550-50).

Fig. 1 details a typical GS experimental scheme, where a laser diode is biased with $I_{\text {bias }}$, and modulated at a frequency $f_{\mathrm{LO}}$ with a modulation depth $P_{m}$, being these the three control parameters of the pulsed regime for a given device. By changing these parameters, the pulses at the output change in width, power, repetition rate and shape.

This temporal pulsed regime has a translation in the frequency domain, where several modes separated by the repetition rate frequency appear around the original modes of the diode laser under GS in a comb-like optical spectrum. This can be also observed in the downconverted electrical spectrum recovered by an electronic spectrum analyzer after detection in a fast photodiode.

In order to start the comparison of both schemes for our PLO (linear and GS modulations) the output optical spectra from the

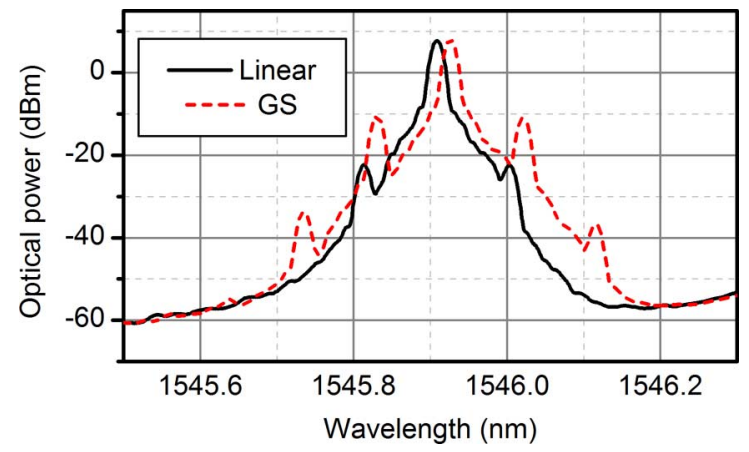

Fig. 2. Optical spectra of the DFB under linear and GS modulation.

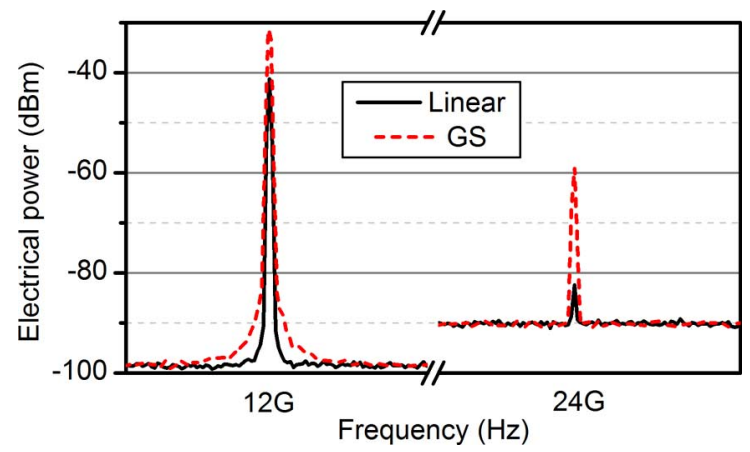

Fig. 3. Electrical spectra of the DFB under linear and GS modulation. The first $(12 \mathrm{GHz})$ and second harmonic $(24 \mathrm{GHz})$ are shown. $\mathrm{RBW}=100 \mathrm{kHz}$, Span $=10 \mathrm{MHz}$.

same DFB laser under both operation modes have been experimentally studied using the same modulation frequency. This is shown in Fig. 2. In order to provide a fair comparison between the two strategies, we fix the same average optical power, $8 \mathrm{dBm}$, in both cases. This value has been chosen in order to ensure adequate linear optical propagation trough an optical fiber [8]. The parameters for GS operation are $I_{\text {bias }}=65 \mathrm{~mA}$, modulation power of $P_{m}=16 \mathrm{dBm}$ and modulation frequency $f_{\mathrm{LO}}=12 \mathrm{GHz}$. For the linear modulation the frequency is also $12 \mathrm{GHz}$, with a $P_{m}=4 \mathrm{dBm}$ which is the maximum value for modulation within the linear region. The $I_{\text {bias }}$ was slightly changed to $61 \mathrm{~mA}$ in order to provide exactly the same average output power as in the GS case $(8 \mathrm{dBm})$ avoiding the addition of extra elements in one of the scenarios such as optical attenuators.

As expected, under GS operation, not only the primary typical side bands corresponding to an amplitude modulated signal appear around the laser main mode as it happens under linear sinusoidal modulation (Fig. 2, continuous line), but also several side modes, separated by the applied modulation frequency $f_{\mathrm{LO}}$, appear (dashed line). The shift towards longer wavelengths is caused by the different bias current. A minor asymmetry is also perceived in the GS optical spectrum, which is in well agreement with the expected behavior under this modulation regime [19].

The outputs from both schemes are downconverted using a photodiode and the output spectra observed in an electric spectral analyzer (ESA). For both cases a main peak appears at the modulation frequency $(12 \mathrm{GHz})$ as well as several high orders harmonics. In Fig. 3 we show a comparison between the 


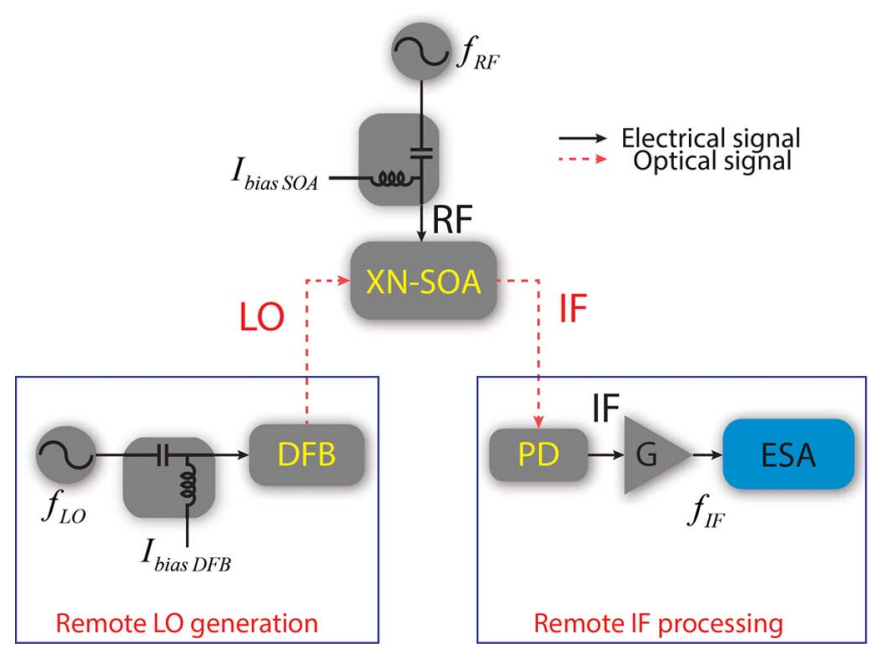

Fig. 4. Experimental setup of the EO receiver based on a XN-SOA using PLO.

fundamental and first harmonic of the GS recovered spectrum (dashed line) and the linearly modulated one (continuous line). The power at the fundamental harmonic is significantly higher for the GS case (around $10 \mathrm{~dB}$ bigger). The power for the second harmonic is also much higher in the GS case as compared to the power of the first harmonic in the linear case, that happens to be very small $(-85 \mathrm{dBm})$. In the GS case, the optical power is related to signal, while in the linear case, a high amount of it is DC optical power not carrying any modulation information.

Hence, though the same optical power is being distributed, its spectral distributions are different. The next section will evaluate the implications of this fact in the performance of these sources as PLO in an EO heterodyning scheme.

\section{Electro-Optical Heterodyne Receiver}

The EO heterodyne receiver under study in the present work is described in Fig. 4. The PLO reaches the optical input of the XN-SOA device (CIP SOA-XN-OEC-1550) where the mixing process takes place. The retrieved IF signal is modulated onto an optical carrier in the same mixing process in the XN-SOA, and is distributed via optical fiber to the Remote IF processing stage (Fig. 4). This IF signal is detected using a high bandwidth photodiode ( $\mathrm{u} 2 \mathrm{t}$ XPV2020R) that performs the optical to electronic conversion and makes the IF electronic signal available [8]. As we can see in this Fig. 4 the RF signal is directly applied to the bias port of the mixing element, the XN-SOA, that comes in a 7-PIN SMA-connector butterfly package with a 50-ohm RF input used to introduce both the bias current and the RF signal to the device (Fig. 4). The special nonlinear characteristics of the XN-SOA element considered offer a high efficient mixing in a compact, polarization independent scheme [8].

\section{EXPERIMENTAL RESULTS}

The scheme of Fig. 4 has been used to evaluate the several figures of merit considered to characterize the performance of the EO heterodyne receiver described previously and the influence of the two PLO schemes (GS and linear modulations). A bias-tee $(65 \mathrm{kHz}-20 \mathrm{GHz}$ bandwidth) allows to introduce the bias current and the direct modulation (linear or GS) to the DFB laser. A bias-tee ( $65 \mathrm{kHz}-20 \mathrm{GHz}$ bandwidth) is also employed in the XN-SOA to introduce the bias current and bias modulation (i.e., RF signal). Both currents are provided by two laser diode drivers. In the remote IF processing stage, a high bandwidth photodiode ( $\mathrm{u} 2 \mathrm{t}$ XPV2020R, $50 \mathrm{GHz} 3-\mathrm{dB}$ bandwidth) performs the conversion of the IF signal from the optical to the electrical domain. A low noise 2-40 GHz preamplifier (Cernex CBL02403050-01) is used before detection in an ESA.

As stated previously, the figures of merit under study are the conversion efficiency, the linearity using the 1-dB compression point and the phase noise via the RMS integrated jitter performance of the system. This latter is of major importance since it characterizes the temporal stability of the recovered IF and it is critical in, for example, photonic signal distribution in Radar systems and Optical Transport Networks (OTN) [15].

\section{A. Conversion Efficiency}

The figure of merit considered to evaluate the efficiency of the EO heterodyne receiver is the downconversion ratio, defined for optoelectronics mixers as the ratio between the electrical power of the downconverted signal at $f_{\mathrm{IF}}$ (output of the photodiode) and the electrical power of the RF signal present after mixing at $f_{\mathrm{RF}}$ [11]. Electrical losses and the effect of the auxiliary 2-40 GHz preamplifier (Cernex CBL02403050-01, G in Fig. 4) included in the setup have been calibrated and the reported values are corrected from these effects.

Fig. 5 details the experimental results obtained after the characterization of the conversion ratio of the EO heterodyne receiver for both a linear and GS PLO and for several XN-SOA bias points versus the RF frequency.

When the XN-SOA is biased at the smallest operating current, below its gain threshold (50 mA, Fig. 5), and the GS PLO (red triangles) is considered, proper mixing behavior can be observed for almost the whole range of RF frequencies under study ( $5 \mathrm{GHz}$ to $15 \mathrm{GHz}$ ). The conversion ratio increases from $-29 \mathrm{~dB}$ for $5 \mathrm{GHz}$ to a maximum of $-17 \mathrm{~dB}$ at $11 \mathrm{GHz}$. When using the linearly modulated PLO (black squares), mixing behavior is not achieved for most of the range, and when it is present, the downconversion ratio is as small as $-28 \mathrm{~dB}$ (at $14 \mathrm{GHz}$ ).

When the $\mathrm{XN}-\mathrm{SOA}$ is biased at higher operating current (150 $\mathrm{mA})$, the mixer performance improves and the heterodyning is observable for the whole RF range and for the two PLO considered. If this operating current is increased even further, the device conversion efficiency somehow stabilizes above $250 \mathrm{~mA}$, being always higher for the GS LO in approximately 11-12 dB. It can be appreciated that as the XN-SOA current increases, the conversion ratios at lower RF frequencies (below $10 \mathrm{GHz}$ ) have a slightly greater increment than for frequencies higher than $10 \mathrm{GHz}$. When the XN-SOA is biased at $450 \mathrm{~mA}$, the GS LO offers a maximum of $-15 \mathrm{~dB}$ conversion efficiency at $8 \mathrm{GHz}$, and it shows a very stable behavior for the whole RF range, except for the value at $12 \mathrm{GHz}$. This is an expected result as $12 \mathrm{GHz}$ is the frequency of the PLO signal and for this IF frequency (RF frequency slightly detuned to $11.9 \mathrm{GHz}$, i.e., IF frequency is $100 \mathrm{MHz}$ ) the RF preamplifier ( $\mathrm{G}$ in Fig. 4) is out of its frequency working range $(2-40 \mathrm{GHz})$. In the view of these results, it can be clearly seen that there is an 11-12 dB 


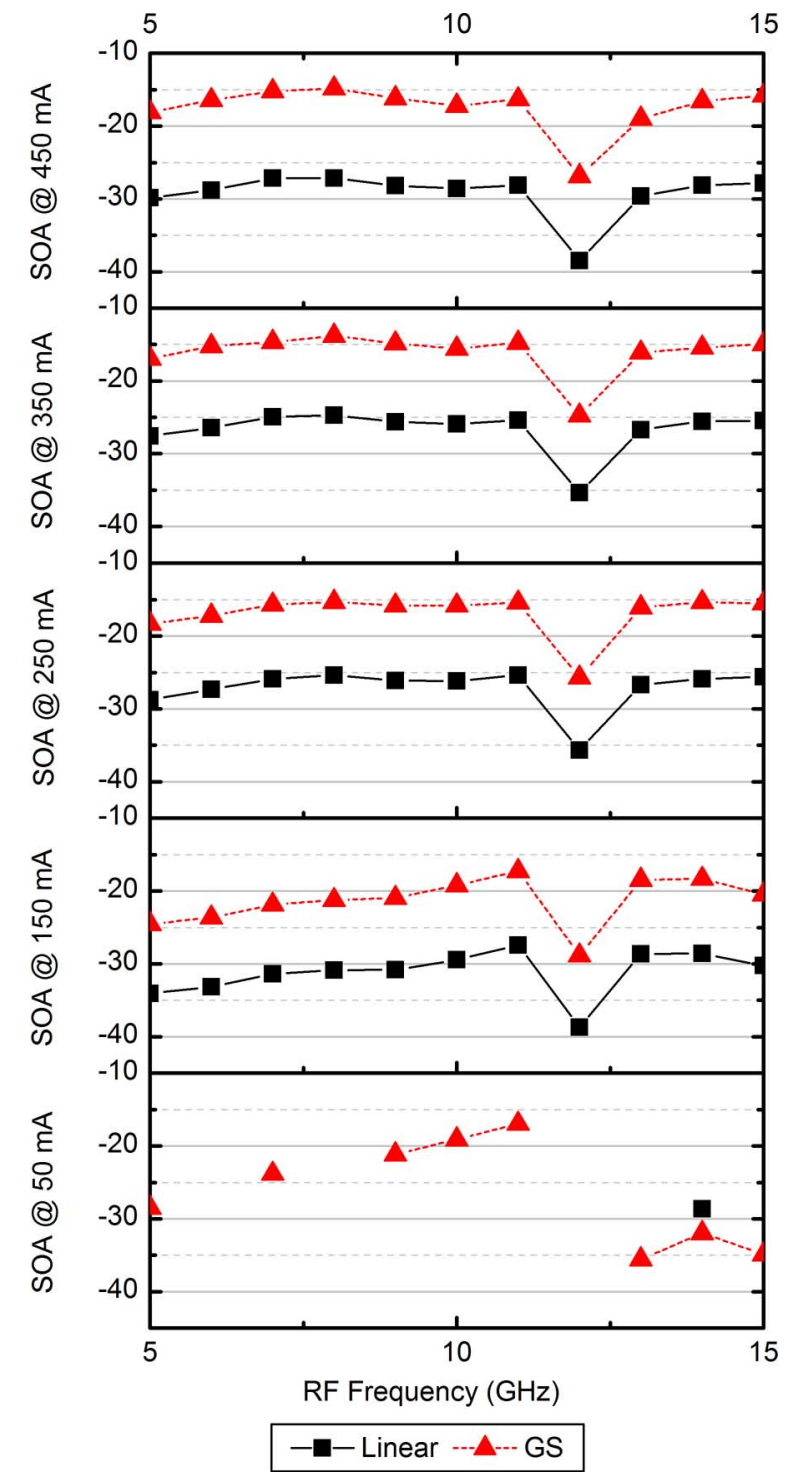

Fig. 5. Downconversion ratio (dB) for both linearly modulated (black squares) and GS modulated (red triangles) PLO under different bias point of the $\mathrm{XN}-\mathrm{SOA}$.

difference between the conversion ratios of both PLO schemes. This corresponds to the difference in modulation power that is applied between the two PLO schemes to guarantee that they emit the same average power. Under these conditions, the GS PLO is able to transport much more LO modulation information under the same average optical power. This higher LO power is transferred to the mixing process in a more efficient way.

\section{B. Linearity}

The linearity of the system has been studied through the 1-dB compression point. In order to do so, the power of the LO is kept constant while the power of the RF signal is increased. The input RF power for which the recovered IF signal did not increase linearly with the power of this RF signal by $1 \mathrm{~dB}$, was identified as the 1-dB compression point.

The RF frequency was swept from $5 \mathrm{GHz}$ to $15 \mathrm{GHz}$ and its power from $1 \mathrm{dBm}$ to $16 \mathrm{dBm}$, as $16 \mathrm{dBm}$ is the maximum input power specified by the manufacturer for the RF port of the XN-SOA. The $1 \mathrm{~dB}$ compression point was not observable, so, within the range considered, the system is linear for all the bias operating points studied for the SOA mixer $(50,150,250$, 350 and $450 \mathrm{~mA}$ ) and for the two types of PLO under evaluation (linearly and GS modulated), being the maximum RF input power specifications of the $\mathrm{XN}-\mathrm{SOA}$ the limiting factor.

\section{Noise: RMS Integrated Timing Jitter}

In order to analyze the noise added by the complete EO receiver (i.e., PLO, XN-SOA and IF retrieving stage) in the downconversion process, the phase noise is evaluated through the RMS integrated jitter. The analysis of this figure of merit is important in order to evaluate the influence of the additional nonlinearities added by the GS process. The well-known standard defining OTN, ITU-T G.709 [14], is used as a reference of an actual potential application.

OTN is the current standard for optical networks with high capacity. It is based in the experience with SONET/SDH networks and was the result of the increasing need of higher line rates, new features such as optical channel management and Forward Error Correction (FEC), and global orientation [20].

There exist three different line rates in OTN: OTN1 (2.66 Gbps), OTN2 (10.7 Gbps) and OTN3 (40 Gbps). Given the operation bandwidth of our EO receiver $(5-15 \mathrm{GHz})$, OTN2 frame is chosen as a restrictive limit to evaluate our noise results. The jitter requirements for OTN interfaces are defined in ITU-T G.8251 [15]. Wideband integration range is chosen instead of narrowband in order to provide a more complete view of eventual spurious signals far from the carrier. In OTN2, the wide band is defined from $20 \mathrm{kHz}$ to $80 \mathrm{MHz}$ [15], and this is the integration range used in our jitter analysis. The maximum allowed RMS integrated jitter in this band in order to ensure proper OTN operation is $49.93 \mathrm{ps}$. This reference value is shown in all the graphs of Fig. 6 as a dashed line.

Fig. 6 shows the RMS integrated jitter measurements for the bias current conditions of the $\mathrm{XN}-\mathrm{SOA}$ previously considered with the exception of that below threshold $(150,250,350$ and $450 \mathrm{~mA}$ ) and for a frequency range from 5 to $11 \mathrm{GHz}$. No data is shown above $12 \mathrm{GHz}$ as the detected Signal to Noise Ratio (SNR) was not enough for the phase noise analyzer to perform a proper phase noise analysis. Following the trend of the analysis of other figures of merit, the EO receiver using a linearly modulated PLO only works properly for the whole frequency range at higher bias current of the XN-SOA (above $350 \mathrm{~mA}$ ). Nevertheless, the EO mixer works properly for GS PLO for all the experimental conditions studied in this analysis and it is important to note that in all cases, the measured jitter is almost one order of magnitude better than in the case of the linearly modulated PLO. This is a significant result as it demonstrates that the use of a GS PLO, that includes some non-linear nature in its generation [12], does not degrade the noise performance of the system.

As the RMS integrated jitter covers a wide frequency range, it includes both the low frequency offset noise (mainly due to phase noise), and the high frequency offset noise (interpreted as floor noise, i.e., Noise Figure). The improvement on the noise performance when using a GS PLO instead of a linearly modulated one has its origin in the fact that under GS regime, a 


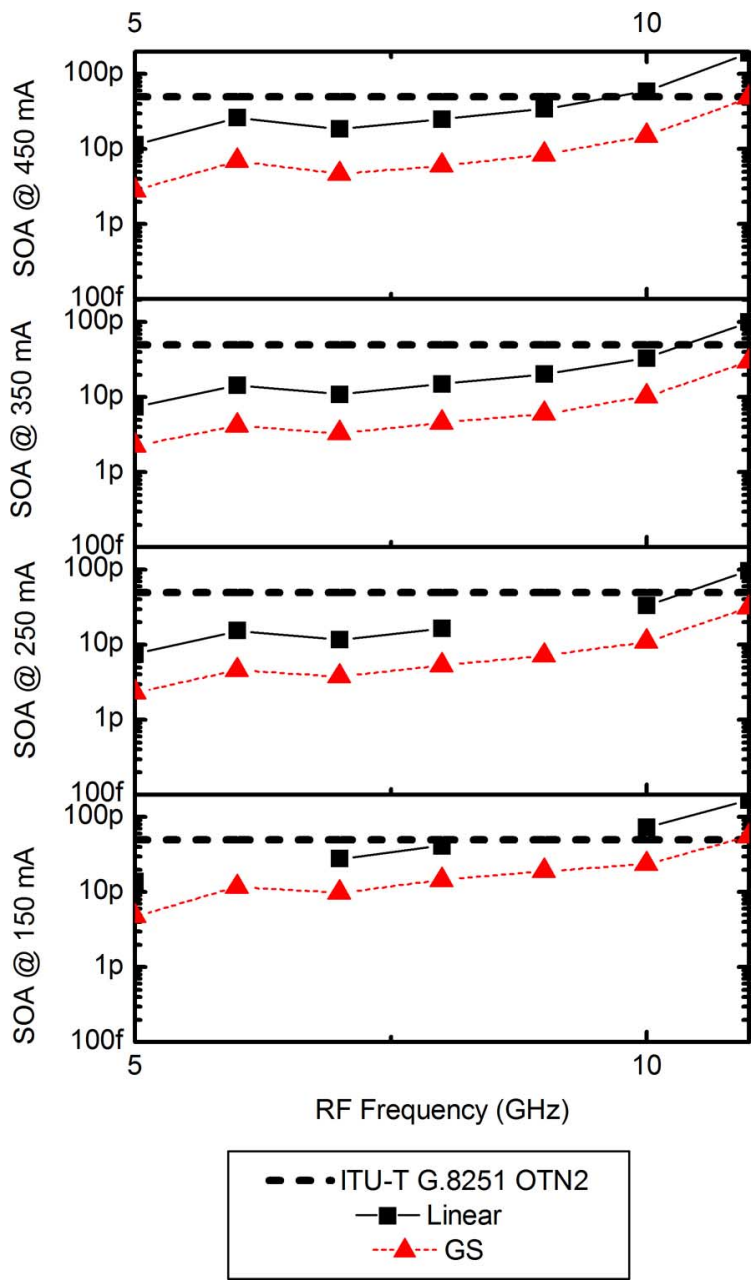

Fig. 6. RMS jitter (s) integrated over the band $20 \mathrm{kHz}-80 \mathrm{MHz}$ (OTN2 protocol) for both linearly modulated (black squares) and GS modulated (red triangles) PLO under different bias point of the XN-SOA.

much higher modulation current is applied to the laser. At the receiver, this results in a higher SNR, or equivalently a better Noise Figure of the system that is directly translated into a better noise performance, especially at higher frequency offsets.

Finally, it is worth mentioning that for the GS PLO, the RMS integrated jitter requirements for OTN2 are met for all the analyzed cases, while the linearly modulated PLO fails to do it for $\mathrm{RF}$ frequencies above $9 \mathrm{GHz}$ for the smaller bias current $(150$ $\mathrm{mA}$ ) and above $10-11 \mathrm{GHz}$ for higher bias current applied to the $\mathrm{XN}-\mathrm{SOA}$ (above $250 \mathrm{~mA}$ ).

\section{CONCLUSION}

We have evaluated in detail the performance of an Electro-Optical (EO) heterodyne receiver based on an Ultra Nonlinear SOA (XN-SOA) considering several figures of merit. The scheme shows moderate conversion ratios, good linearity and a reduced root mean square (RMS) integrated jitter. However, its behavior can be significantly improved when the Photonic LO is based on a pulsed optical source as a Gain Switching (GS) DFB diode laser instead of the traditionally employed linearly modulated DFB. This pulsed regime can be induced in any diode laser, even commercially available, and permits a more efficient distribution of the same amount of optical power, where the energy transmitted is related to the LO modulation signal rather than lost in DC optical power. The performance of the receiver improves for all the figures of merit considered. The downconversion ratio increases by $11-12 \mathrm{~dB}$ for all RF frequency values considered and the several XN-SOA working points under study. The RMS integrated jitter is reduced by one order of magnitude, showing values that are well below the jitter requirements of ITU-T G.709 OTN standard [14] for the whole RF frequency range. The reported EO heterodyne receiver, along with the use of a Photonic LO based on GS allows for a cost-effective, compact, polarization-independent and scalable solution with enhanced performance for applications requiring LO and IF remote distribution.

\section{REFERENCES}

[1] A. J. Seeds and K. J. Williams, "Microwave photonics," J. Lightw. Technol., vol. 24, no. 12, pp. 4628-4641, 2006.

[2] J. Marti and J. Capmany, "Microwave photonics and radio-over-fiber research," IEEE Microw. Mag., vol. 10, pp. 96-105, Jun. 2009, no..

[3] P. Berger, J. Bourderionnet, F. Bretenaker, D. Dolfi, and M. Alouini, "Time delay generation at high frequency using SOA based slow and fast light," Opt. Exp., vol. 19, no. 22, pp. 21180-21188, Oct. 2011.

[4] T. Kuri, H. Toda, and K. Kitayama, "Dense wavelength-division multiplexing millimeter-wave-band radio-on-fiber signal transmission with photonic downconversion," J. Lightw. Technol., vol. 21, no. 6, pp. 1510-1517, Jun. 2003.

[5] B. Hraimel, "Photonic down-conversion of millimeter wave multiband orthogonal frequency division multiplexing ultra-wideband using four wave mixing in an electro-absorption modulator," J. Lightw. Technol., vol. 28, no. 13, pp. 1987-1993, Jul. 2010.

[6] P. Acedo, H. Lamela, and C. Roda, "Optoelectronic up-conversion using compact laterally mode-locked diode lasers," IEEE Photon. Technol. Lett., vol. 18, no. 17-20, pp. 1888-1890, 2006.

[7] N. Breuil, M. Dispenza, L. Morvan, A. M. Fiorello, S. Tonda, D. Dolfi, M. Varasi, and J. Chazelas, "New optical modulation schemes applied to local oscillator distribution in radar systems," in Proc. MWP, 2004, pp. $119-122$

[8] Á. R. Criado, C. de Dios, and P. Acedo, "Characterization of ultra-nonlinear SOA in a heterodyne detector configuration with remote photonic local oscillator distribution," IEEE Photon. Technol. Lett., vol. 24, no. 13, pp. 1136-1138, Jul. 2012.

[9] J. Palací, G. Villanueva, and J. Herrera, "EAM-SOA millimeter-wave frequency up-converter for radio-over-fiber applications," Opt. Commun., vol. 284, no. 1, pp. 98-102, Jan. 2011.

[10] C. Bohemond, A. Sharaiha, T. Rampone, and H. Khaleghi, "Electrooptical radiofrequency mixer based on semiconductor optical amplifier," Electron. Lett., vol. 47, no. 5, pp. 331-333, 2011.

[11] S. Jun-Hyuk, C. Chang-Soon, K. Young-Shik, C. Yong-Duck, K. Jeha, C. Woo-Young, J. H. Seo, C. S. Choi, Y. S. Kang, Y. D. Chung, J. Kim, and W. Y. Choi, "SOA-EAM frequency up/down-converters for 60-GHz bidirectional radio-on-fiber systems," IEEE Trans. Microw. Theory Tech., vol. 54, no. 2, pp. 959-966, 2006.

[12] P. P. Vasil'ev, I. H. White, and J. Gowar, "Fast phenomena in semiconductor lasers," Reports Progr. Phys., vol. 63, p. 1997, 2000.

[13] S. M. Riecke, H. Wenzel, S. Schwertfeger, K. Lauritsen, K. Paschke, R. Erdmann, and G. Erbert, "Picosecond spectral dynamics of gainswitched DFB lasers," IEEE J. Quantum Electron., vol. 47, no. 5, pp. 715-722, May 2011

[14] ITU-T, Interfaces for the Optical Transport Network (OTN), ITU-T Rec. G.709/Y.1331 2001.

[15] ITU-T, The Control of Jitter and Wander Within the Optical Transport Network (OTN), ITU-T Rec. G.8251 2001.

[16] H. Shams, A. Kaszubowska-Anandarajah, P. Perry, and L. Barry, "Demonstration and optimization of an optical impulse radio ultrawideband distribution system using a gain-switched laser transmitter," J. Opt. Netw., vol. 8, no. 2, p. 179, Jan. 2009 
[17] K. Taira, T. Hashimoto, and H. Yokoyama, "Two-photon fluorescence imaging with a pulse source based on a 980-nm gain-switched laser diode," Opt. Exp., vol. 15, no. 5, p. 2454, Mar. 2007.

[18] H. Liu, C. Gao, J. Tao, W. Zhao, and Y. Wang, "Compact tunable high power picosecond source based on Yb-doped fiber amplification of gain switch laser diode," Opt. Exp., vol. 16, no. 11, pp. 7888-7893, 2008.

[19] C. de Dios and H. Lamela, "Improvements to long-duration low-power gain-switching diode laser pulses using a highly nonlinear optical loop mirror: Theory and experiment," J. Lightw. Technol., vol. 29, no. 5, pp. 700-707, 2011.

[20] Agilent Technologies, Application Note: 1379. An Overview of ITU-T G. 709, 2001 .

C. de Dios received the M.S. degree in applied physics and electronics and the M.Res. in 2004 and Doctorate in 2010 for her work in ultrafast pulsed diode lasers and nonlinear pulse compression, from the Universidad Complutense of Madrid, Madrid, Spain.

She then joined the private sector as a Technical Consultant for HewlettPackard. Since 2002, she has been a member of the Optoelectronics and Laser Technology Group at the Universidad Carlos III de Madrid. Currently, she is an Assistant Professor at the Electronics Technology Department in this same university. Her research interests are high-speed optical communications, pulsed semiconductor laser sources, nonlinear optical phenomena and sub-terahertz and millimeter wave photonic signal synthesis and detection.
A. R. Criado (S'09) received the B.Sc. and M.Sc. (2009) n telecommunication engineering and the M.Res. degree on advanced electronics Systems from Universidad Carlos III de Madrid, Madrid, Spain, in 2011, where he is currently working toward the Ph.D. degree in the field of low noise photonic generation and homodyne/heterodyne detection of mm-waves and sub-THz signals.

$\mathrm{He}$ has been involved in research tasks on laser diagnostics for fusion plasmas, specifically electronic density measurement using two color multichannel laser interferometry, and he worked as collaborator researcher during 2009 and 2010 in the National Fusion Laboratory in CIEMAT (Spain).

E. Prior received the B.Sc. and M.Sc. degrees in telecommunication engineering from Universidad Carlos III de Madrid, Madrid, Spain, in 2012.

Since 2011, she has been involved in research and development tasks related to Microwave Photonics and Radio over Fiber, in both transmission and receiver systems. She currently works on pulsed operation (gain switching) on VCSELs at Universidad Carlos III de Madrid.

P. Acedo (M'99) received the B.S. degree on telecommunication engineering in 1993 from the Universidad Politécnica de Madrid, Madrid, Spain, and the Doctorate (hons) from the Universidad Carlos III de Madrid, Spain, in 2000 for his work on heterodyne two color laser interferometry for fusion plasma diagnostics at the Stellarator TJ-II, (Laboratorio Nacional de Fusión, CIEMAT, Madrid) and Tokamak C-Mod (Plasma Science and Fusion Centre, Massachusetts Institute of Technology)

In 2002 he was appointed as Assistant Professor by Universidad Carlos III de Madrid where he has continued with the development of scientific instrumentation systems for fusion plasma diagnostics and biomedical applications. He has been also involved in research on high speed semiconductor laser devices for microwave and millimeter-wave generation and processing. Currently his interests in this line are the development of integrated active photonic antennas for $\mathrm{mm}$-wave and $\mathrm{THz}$ generation and detection. 